\title{
Polyherbal Formulation (PDBT Capsules) Containing Extracts of Ayurvedic Plants for Treatment of Prediabetes
}

\author{
Pallavi Londhe ${ }^{1}$, Rohini Waghmare ${ }^{1}$, Amit Nakanekar ${ }^{2}$, Kuldip Kohli ${ }^{3}$, Pratima Arun Tatke ${ }^{1, *}$ \\ ${ }^{1}$ Chintaman Uttamdas Shah College of Pharmacy, Shrimati Nathibai Damodar Thakarsey Women's University, Mumbai, India \\ ${ }^{2}$ Ayurvedic Medical College, Nagpur, India \\ ${ }^{3}$ Government of Maharashtra, Mumbai, India
}

\section{Email address:}

pasolo2590@gmail.com (P. Londhe), rohinishyam7@gmail.com (R. Waghmare), amitnakanekar@gmail.com (A. Nakanekar), kohliayurveda@gmail.com (K. Kohli), pratima.tatke@cushahpharmacy.sndt.ac.in (P. A. Tatke), drpratimatatke@gmail.com (P. A. Tatke)

${ }^{*}$ Corresponding author

\section{To cite this article:}

Pallavi Londhe, Rohini Waghmare, Amit Nakanekar, Kuldip Kohli, Pratima Arun Tatke. Polyherbal Formulation (PDBT Capsules) Containing Extracts of Ayurvedic Plants for Treatment of Prediabetes. International Journal of Diabetes and Endocrinology.

Vol. 6, No. 1, 2021, pp. 16-23. doi: 10.11648/j.ijde.20210601.14

Received: December 7, 2020; Accepted: January 6, 2021; Published: January 18, 2021

\begin{abstract}
In Prediabetes blood sugar levels are found to be higher than normal levels. It is considered as pre stage of diabetes as people with prediabetes, if remain undiagnosed, have chances of developing type 2 diabetes. Studies show that if diabetes is treated in the prediabetic stage, it results in complete cure of diabetes. The objective of the present study was to investigate effect of PDBT capsules containing extracts of well-known Ayurvedic plants such as Zingiber officinale, Tinospora cordifolia, Pterocarpus marsupium, Gymnema sylvester, and Momordica charantia on prediabetic condition induced in Wistar rats. High fat fructose diet was fed to male Wistar rats to induce prediabetes. After induction on $11^{\text {th }}$ week of study, the prediabetic rats were divided in different groups. Treatment groups received Pioglitazone (3 mg/kg) (standard control), test formulation, PDBT capsule $(200 \mathrm{mg} / \mathrm{kg}$ ) (test group) along with high fat fructose diet. Normal diet control group received normal diet and high fat fructose diet control group received only high fat fructose diet. The different biochemical parameters, blood glucose levels and body weights were monitored. Animals were sacrificed at the end of study period. Histopathological studies on the major organs such as liver, kidney, pancreas was performed. High fat fructose diet induced of prediabetes in rats. Significant decrease in levels of fasting blood glucose and biochemical parameters in treatment groups was observed. The histopathological study showed no abnormalities. The present research show that PDBT capsules can be efficiently used in prediabetes treatment. Hence, PDBT capsule can be a potential agent for the treatment of prediabetes.
\end{abstract}

Keywords: Prediabetes, Zingiber officinale, Tinospora cordifolia, Pterocarpus marsupium, Gymnema sylvester, Momordica charantia

\section{Introduction}

Diabetes mellitus is a cluster of different disorders. It is diagnosed by hyperglycemia and glucose intolerance. In diabetes there may be lack of insulin or action of insulin might be defective or both [1].

Diabetes mellitus has affected more than 170 million individuals worldwide [2]. In India the diabetes is one of the major health problem. In the treatment of the diabetes, new area for the research has been developed, that is to treat diabetes in its primary stage $[3,4]$. The condition is commonly known as prediabetes. As per Tabák A et al Prediabetes, typically defined as blood glucose concentrations higher than normal, but lower than diabetes thresholds, is a high-risk state for diabetes development $[3,5]$.

In prediabetes the fasting glucose levels are between 100 and $125 \mathrm{mg} / \mathrm{dl}$ and while in oral glucose tolerance test (OGTT) glucose levels are between 140 and $199 \mathrm{mg} / \mathrm{dl}$. [6, 7].

Prediabetes is becoming more common in the South Asian population (both in India and abroad). As per the International Diabetes Federation reports, 280 million individuals were pre-diabetic in year 2011. It has been proposed that 398 million individuals having prediabetes are 
likely to develop type 2 diabetes if remain untreated. [8]. International Diabetes Federation indicates that approximately 471 million may have prediabetes by 2035 .

According to WHO, if people have one of two distinct states: impaired fasting glucose (IFG), or impaired glucose tolerance (IGT), then they are at high risk of developing diabetes [9].

It is proved that type 2 diabetes can be cured when treated in its prediabetic stage [3]. Lifestyle modification can reduce $40-70 \%$ relative-risk and help in diabetes prevention. Pharmacological interventions are also found useful in clinical studies [10].

Oral hypoglycemic agents help to maintain the blood glucose level but they show several unwanted consequences like weight gain, hypoglycemia, insulin resistance, complications of cardiovascular system and gastrointestinal disorders [11]. Pathogenesis of diabetes mellitus involves multiple factors. Hence the drugs which can target at multiple receptors, and which are safe, cheap and easily available are the need of the time. The traditional Ayurvedic drug treatments offer good therapeutic results [12]. In Ayurvedic literature several medicinal herbs are suggested for the treatment of diabetes mellitus [13]. Traditional medicinal plants and traditional formulation have potent phytochemicals are helpful in developing new types of therapy. Potential phytochemicals from Medicinal plants show multiple beneficial effects in treatment of diabetes as well as diabetes-related complications $[14,15]$.

The scientific validation of pharmacological effects and phytochemicals in Indian medicinal plants with modern technique is very limited. A large number of plants used with enormous potential are yet to be validated scientifically and systematically for their pharmacological activity. The systematic evaluation and information on the evidence-base of these plants will enhance the global acceptance about Ayurveda and Indian herbals $[14,16]$.

In the present study therefore attempts were made for formulation and evaluation of poly herbal capsules (PDBT capsules) against the prediabetic conditions induced by high fat fructose diet in Wistar strain rats.

Poly-herbal formulations have diversity of active principles and hence they have potent effect. These actives may give synergistic effect. PDBT capsules contain aqueous extracts of Zingiber officinale, Tinospora cordifolia, Pterocarpus marsupium, Gymnemma sylvestre and Momordica charantia in equal quantity. Previous studies of these plants were carried out by different authors for proving the actions of individual drug for its safety and antidiabetic efficacy studies [15, 17-20]. Here in this study we have evaluated the combination of these medicinal plants against prediabetic condition in rats.

\section{Materials and Methods}

\subsection{Chemicals}

PDBT capsules were procured from Podar Ayurved College Mumabi. Pioglitazone $(5 \mathrm{mg})$ by Aventis Pharma were purchased from local market.

Ready to use biochemical kits for different parameters were purchased ARK diagnostics. The different blood parameters such as total cholesterol (TC), triglyceride (TG), high-density lipoprotein (HDL), alanine aminotransferase (ALT), alkaline phosphatase (ALP) for liver tests serum creatinine and blood urea nitrogen were evaluated using these kits. For estimation of all biochemical parameters automated bioanalyzer (ChemWell) was used.

\subsection{Experimental Animals}

Current study was conducted as per prior approval from the Institutional Animal Ethics Committee of C. U. Shah College of pharmacy SNDT University (IAEC approval number is CUSCP/IAEC/31/2012-2013).

Wistar rats of both sexes, weighing 180-210 g were procured from Bharat Serum and Vaccine Pvt. Ltd., Mumbai, India, Animals were provided balanced laboratory diet and given clean water ad libitum. They were kept at $20 \pm 2{ }^{\circ} \mathrm{C}, 65-$ $70 \%$ humidity, and day/night cycle $(12 \mathrm{~h} / 12 \mathrm{~h})$.

\subsection{Experimental Design}

\subsubsection{Induction of Prediabetes}

Prediabetes was induced in animals by orally feeding High fat fructose Diet to animals for 15 weeks. The high-fat diet comprises approximately $21 \%$ protein, $66.5 \%$ fat, $12.5 \%$ carbohydrates. The fasting blood glucose level and oral glucose tolerance test (OGTT) were estimated. [21] The rats showing fasting glucose level between 100 and $125 \mathrm{mg} / \mathrm{dl}$ and glucose level between 140 and $199 \mathrm{mg} / \mathrm{dl}$ in OGTT test were considered as prediabetic $[6,7,22]$ and selected for the study. The animals were then randomized into four groups ( $n=6 /$ group). The drug treatment was given for 4 weeks. During the treatment the animals were divided in following groups.

Group 1: Normal diet control group, this group received normal diet throughout the study and received vehicle $0.1 \%$ Sodium Carboxy methyl cellulose ( $\mathrm{Na} \mathrm{CMC}$ ) during the intervention.

Group 2: High fat fructose diet control group (HFD group) received High fat fructose diet only and no treatment.

Group 3: Standard control group received treatment of Pioglitazone $(3 \mathrm{mg} / \mathrm{kg})$ with high fat fructose diet during intervention.

Group 4: PDBT treatment group received treatment of PDBT capsules $(200 \mathrm{mg} / \mathrm{kg})$ during intervention and high fat fructose diet throughout the study.

Fasting blood glucose levels were estimated weekly using one touch electronic glucometer and glucose test strips. To evaluate the other biochemical parameters, blood samples were withdrawn from the retro orbital plexus of the animals after overnight fasting. Serum was separated and used for estimating various biochemical parameters. All biochemical parameters were estimated at different intervals, before induction to find basal values while every month during induction as well as twice during drug intervention. The 
animals were sacrificed at the end of 4 weeks of intervention and vital organs viz. kidney, liver, pancreas were isolated for histopathological study.

\subsubsection{Statistical Analysis}

All the values of in this study were expressed as mean \pm standard error of mean (S. E. M). The results were analysed for statistics using InStat software. All results were analysed by one way ANOVA and Dunnet's t-test.

\section{Results}

\subsection{Effect of PDBT Capsules on Different Biochemical Parameters}

\subsubsection{Effect of PDBT Capsules on Fasting Blood Glucose Levels}

Levels of fasting blood glucose in all groups are shown in Figure 1. There was an age-related progressive increase in fasting blood glucose levels in the vehicle control group. Feeding of high fat fructose diet in the HFD group caused increase in fasting blood levels. Compared to vehicle control, there was a significant increase in the fasting blood glucose values at the $24^{\text {th }}$ and $30^{\text {th }}$ day. On $30^{\text {th }}$ day $25 \%$ increase in fasting glucose levels were found as compared to normal control group. In the treatment groups, there was a significant decrease in fasting glucose levels on $16^{\text {th }}$ day, $24^{\text {th }}$ day, $30^{\text {th }}$ day of drug treatment. Treatment of PDBT formulation and pioglitazone reduced fasting glucose levels of prediabetic rats upto $84 \pm 0.72$ $\mathrm{mg} / \mathrm{dL}$ and $87 \pm 0.8 \mathrm{mg} / \mathrm{dL}$ respectively on $30^{\text {th }}$ day of intervention. However, there is no statistically significant difference in glucose levels between the pioglitazone and PDBT capsule treated groups.

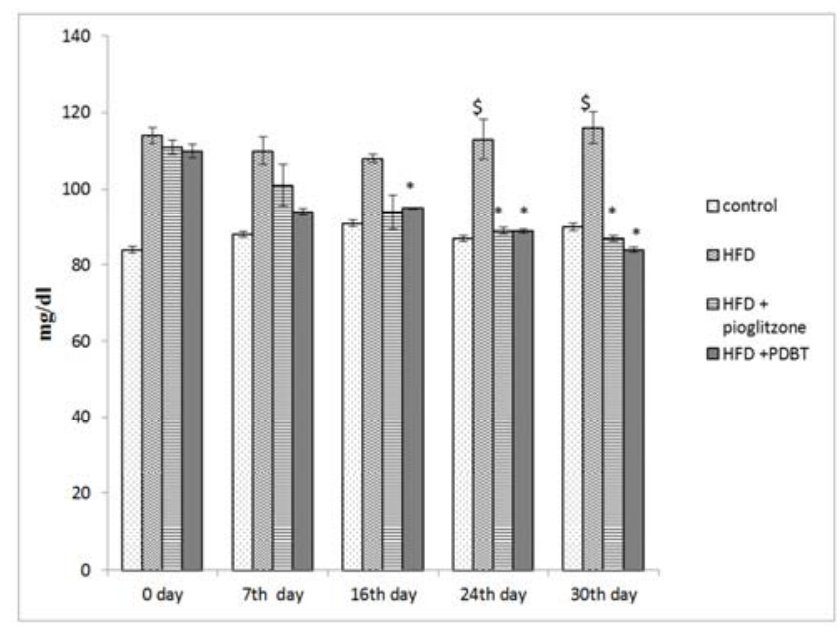

Figure 1. Effect of PDBT capsules on fasting blood glucose levels of rats.

Values are expressed as mean \pm SEM., $n=6 ; n=$ Number of animals.

* Significant decrease when compared with high fat fructose diet control group, $\mathrm{p}<0.01$

$\$$ Significant increase when compared with control group, $\mathrm{p}<0.05$

Control: vehicle control, HFD: High fat fructose diet control

\subsubsection{Effect of PDBT Capsules on Body Weight of Rats}

Changes in body weight in all groups are shown in Figure 2. All groups showed age-related increase in body weight. The prediabetic control rats showed statistically significant increase in body weight with prediabetes symptoms like polyuria, polydipsia, polyphagia indicating that prediabetic condition was induced.

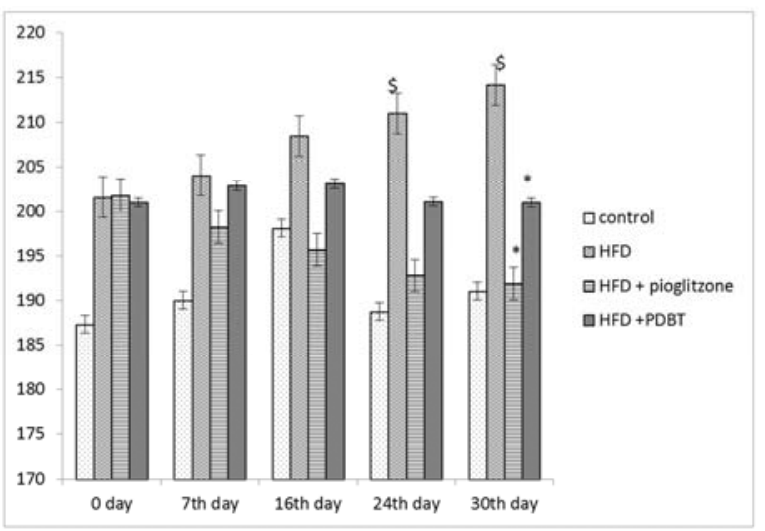

Figure 2. Effect on body weight of rats treated with PDBT capsules

Values are expressed as mean \pm SEM., $n=6 ; n=$ Number of animals. * Significant decrease when compared with high fat fructose diet group, $\mathrm{p}<0.05$ $\$$ Significant increase when compared with control group, $\mathrm{p}<0.05$ Control: vehicle control, HFD: High fat fructose diet control

The body weights of animals treated with formulations was significantly decreased as compared with HFD control group. At the $30^{\text {th }}$ day of intervention pioglitazone and PDBT formulation treatment reduced weight to $191.91 \pm 6.26$ gram and $201 \pm 6.37$ gram respectively. The results indicate that the PDBT capsules were effective in controlling the obesity associated with prediabetic condition.

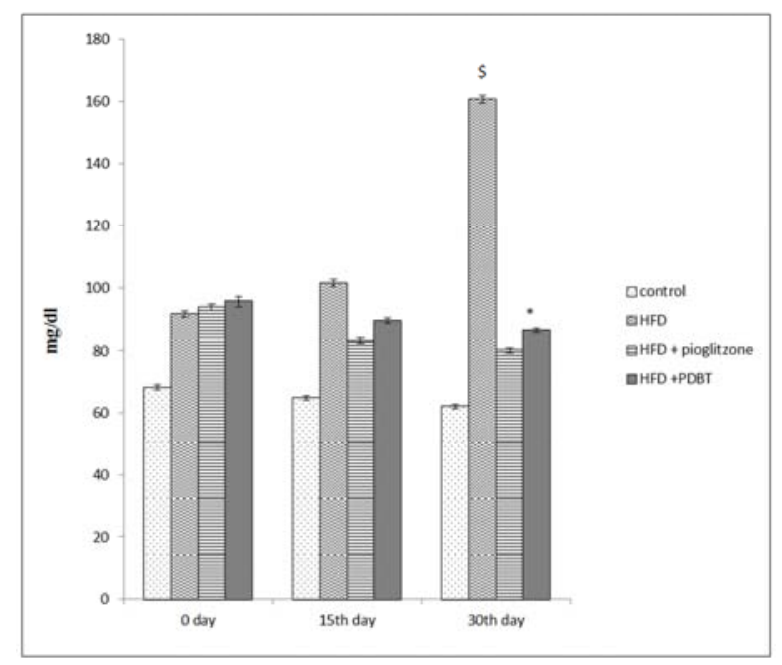

Figure 3. Effect on Cholesterol levels in prediabetic rats treated with PDBT capsule.

Values are expressed as mean \pm SEM., $n=6 ; n=$ Number of animals.

* Significant decrease when compared with high fat fructose diet group, $\mathrm{p}<0.05$

$\$$ Significant increase when compared with control group, $\mathrm{p}<0.05$

Control: vehicle control, HFD: High fat fructose diet control 


\subsubsection{Effect of PDBT Capsules on Lipid Profile of Rats}

\section{i. Effect on Serum Total Cholesterol Levels}

Serum total cholesterol levels in all groups are shown in Figure 3 . The mean total cholesterol levels were significantly increased in prediabetic control animals when compared with vehicle control rats. Formulations treatment showed statistically significant decrease in the total cholesterol levels. Decrease in elevated levels with the standard drug (Pioglitazone) treatment was found to be more $(80.16 \pm 0.76 \mathrm{mg} / \mathrm{dL})$ as compared to PDBT formulation $(86.27 \pm 0.74 \mathrm{mg} / \mathrm{dL})$. Results indicate that the formulations are effective in the elevated total cholesterol levels.

\section{ii. Effect on Serum Triglycerides Levels}

Figure 4 shows the serum triglycerides levels in all groups. There was a significant increase in the triglyceride levels on the $15^{\text {th }}$ day and $30^{\text {th }}$ day in prediabetes control rats as compared to normal rats. The triglyceride levels in rats treated with formulation significantly decreased on $15^{\text {th }}$ and $30^{\text {th }}$ day of drug treatment when compared with prediabetes control animals.

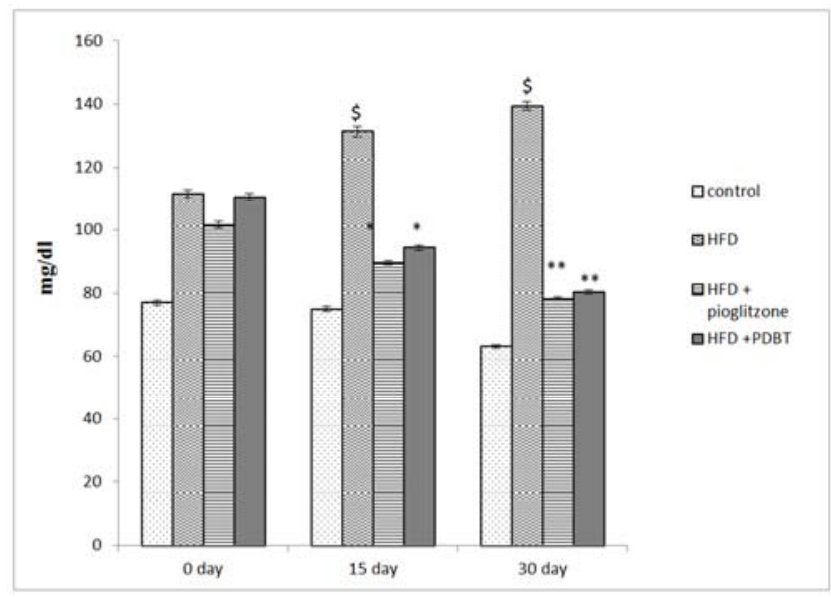

Figure 4. Effect on triglycerides levels in prediabetic rats treated with PDBT capsules.

\$ Significant increase when compared with control group, $\mathrm{p}<0.05$

* * Significantly decrease when compared with high fat fructose diet group, $\mathrm{p}<0.01$

* Significantly decrease when compared with high fat fructose diet group, $\mathrm{p}<0.05$

Control: vehicle control, HFD: High fat fructose diet control

Standard drug Pioglitazone reduced elevated triglyceride levels to $78.29 \pm 0.61 \mathrm{mg} / \mathrm{dL}$ while PDBT formulation treatment reduced elevated triglyceride levels to $80.36 \pm 0.70 \mathrm{mg} / \mathrm{dL}$.

However the decrease in triglyceride levels of treatment groups PDBT capsules and standard drug (Pioglitazone) is not statistically different. The results indicate that, PDBT capsules was effective in controlling the lipid levels.

\section{iii. Effect on HDL Levels}

The HDL levels are shown for all groups in Figure 5. It is observed that oral feeding high fat fructose diet caused elevation in HDL levels. It was also found that the vehicle control group and treatment groups are showing decrease in
HDL levels in rats during the treatment. On $30^{\text {th }}$ day the decrease was statistically significant as compared to High fat fructose diet control group and found to be $45.92 \pm 0.65$ $\mathrm{mg} / \mathrm{dL}$ for standard control group while $46.41 \pm 0.71 \mathrm{mg} / \mathrm{dL}$ for PDBT capsule group.

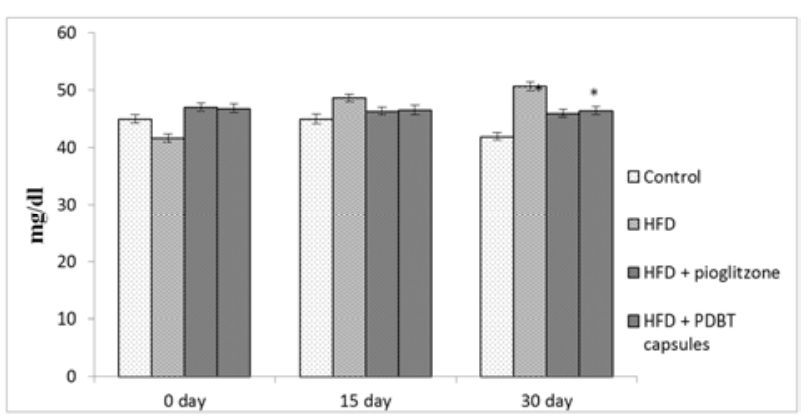

Figure 5. Effect on HDL levels in prediabetic rats treated with PDBT capsules.

* Significant decrease when compared with high fat fructose diet group, $\mathrm{p}<0.05$ Control: vehicle control, HFD: High fat fructose diet control

\subsubsection{Effect of PDBT Capsules on Liver Biomarker of Prediabetic Rats}

\section{i. Effect on AST Levels}

Levels of AST are given in figure 6. Prediabetes caused increase in AST levels in HFD control as well as treatment groups. On $30^{\text {th }}$ day of drug intervention decrease in AST level with PDBT capsule treatment $(36.07 \pm 0.12 \mathrm{U} / \mathrm{L})$ as well as pioglitazone treatment $(30.31 \pm 0.14 \mathrm{U} / \mathrm{L})$ was found. However the decrease is not statistically significant and not different from each other groups.

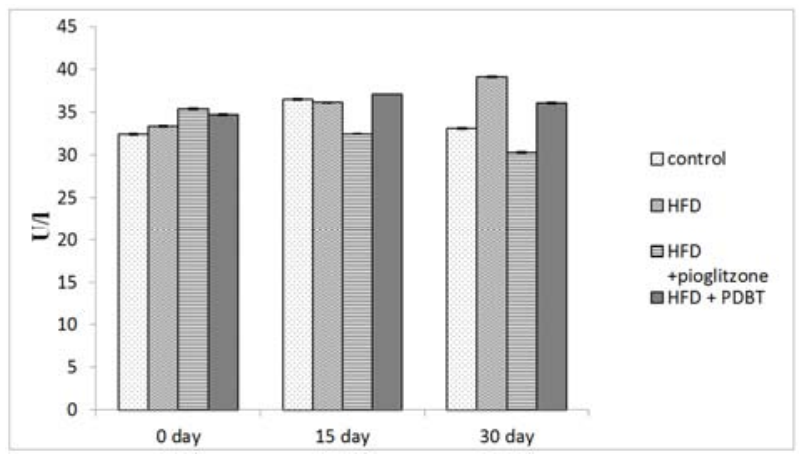

Control: vehicle control, HFD: High fat fructose diet control

Figure 6. Effect on AST levels in prediabetic rats treated with PDBT capsule.

\section{ii. Effect on ALT Levels}

The ALT levels in all groups are shown in figure 7. Increase in ALT levels were found with administration of high fat fructose diet. The prediabetic animals treated PDBT capsules and standard drug showed decrease in ALT levels to $38.15 \pm 0.15 \mathrm{U} / \mathrm{L}$ and $36.51 \pm 0.15 \mathrm{U} / \mathrm{L}$ respectively. However the decrease is not statistically significant.

The decrease in liver marker levels AST and ALT in the prediabetic rats treated with PDBT capsule indicating that the formulation is hepatoprotective. 


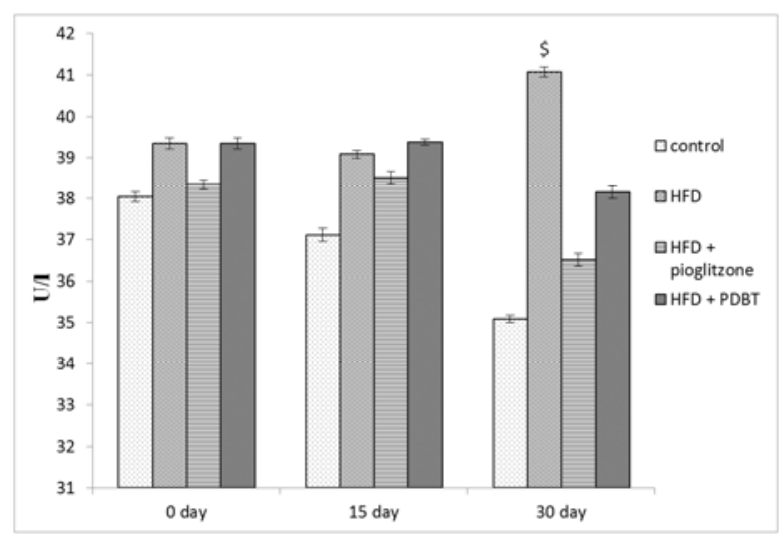

Figure 7. Effect on ALT levels in prediabetic rats treated with PDBT capsules.

$\$$ Significant increase when compared with control group, $\mathrm{p}<0.05$

Control: vehicle control, HFD: High fat fructose diet control

\subsubsection{Effect on Kidney Parameters}

\section{i. Effect on Serum Creatinine Levels}

The serum creatinine levels are shown in figure 8. Prediabetic animals treated with PDBT capsules and standard drug (Pioglitazone) showed decrease in the serum creatinine levels $0.8 \pm 0.09 \mathrm{mg} / \mathrm{dL}$ and $0.8 \pm 0.07 \mathrm{mg} / \mathrm{dL}$ respectively, when compared with prediabetic control animals. PDBT capsules treatment decreased serum creatinine level same as that of standard drug treatment group

Decrease in the Creatinine levels of prediabetic animals indicates that the capsules are nephroprotective.

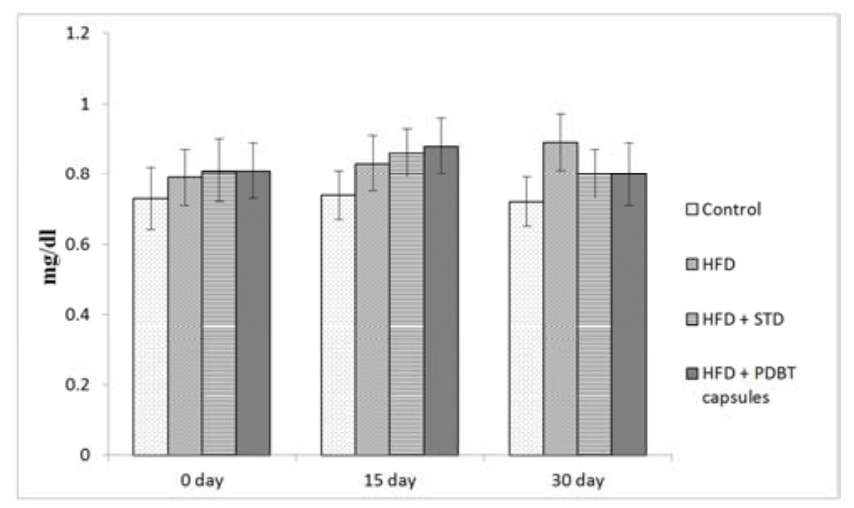

Control: vehicle control, HFD: High fat fructose diet control STD: Pioglitazone

Figure 8. Effect on Creatinine levels in prediabetic rats treated with PDBT capsules.

\section{ii. Effect on Blood Urea Nitrogen Levels}

Blood urea nitrogen levels are shown in figure 9. There was rise in blood urea nitrogen level found in prediabetic control rats when compared with normal rats. PDBT capsule treatment showed decrease in the blood urea nitrogen $18.47 \pm 0.09 \mathrm{mg} / \mathrm{dL}$ when compared with prediabetic treated rats, the same action showed in case of standard drug $(18.38 \pm 0.07 \mathrm{mg} / \mathrm{dL})$. The results indicate that the PDBT capsules have therapeutic activity same as that of standard drug (Pioglitazone).

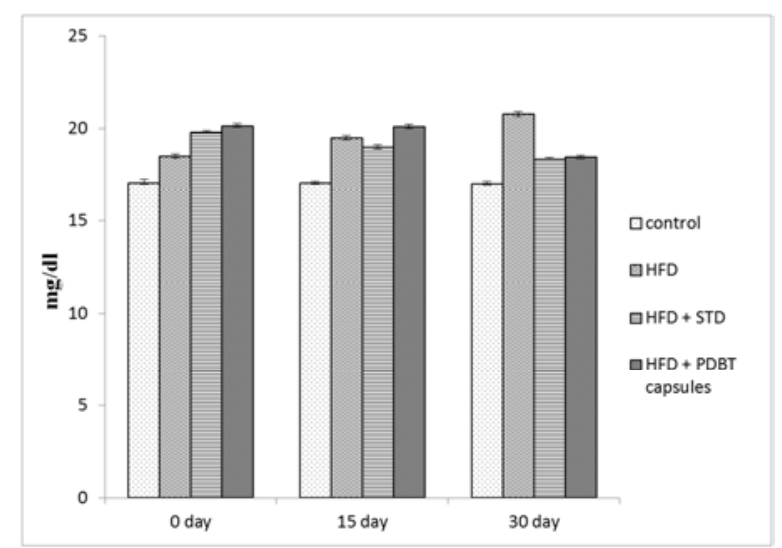

Control: vehicle control, HFD: High fat fructose diet control STD: Pioglitazone

Figure 9. Effect on blood urea nitrogen levels in prediabetic rats treated with PDBT capsules.

\subsection{Results of Histopathology}

At the end of intervention the animals were sacrificed. The vital organs were removed and fixed in $10 \%$ formalin, and subjected to histopathological study.

\subsubsection{Pancreas}

In normal control rats many islets were evenly distributed throughout the cytoplasm of the pancreas (Figure 10-a). The prediabetic control rats (Figure 10-b) and prediabetic rats treated with pioglitazone (Figure 10-c), pancreas showed no any degeneration and necrosis of islet of Langerhans. The prediabetic rats treated with PDBT capsules (Figure 10-d) do not showed abnormal structure of islets.

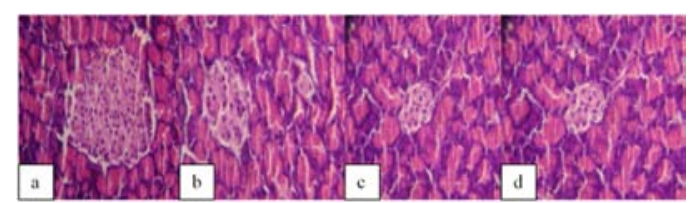

Figure 10. Histopathology study of pancreas of prediabetic rats treated with PDBT capsules.

10a normal control rats, $10 \mathrm{~b}$ prediabetic control rats, $10 \mathrm{c}$ prediabetic rats treated with pioglitazone, $10 \mathrm{~d}$ prediabetic rats treated with PDBT capsule

\subsubsection{Liver}

The liver of normal rats (Figure 11-a), prediabetic control rats (Figure 11-b) and standard drug (Pioglitazone) treated rats (Figure 11-c) appeared normal in histopathological studies. In prediabetic rats treated with PDBT capsules (Figure 11-d) does not showed any granular degeneration.

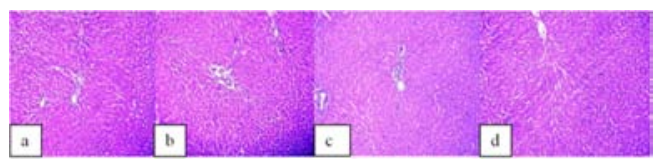

Figure 11. Histopathology study of liver of prediabetic rats treated with PDBT capsules.

11a normal control rats, $11 \mathrm{~b}$ prediabetic control rats, $11 \mathrm{c}$ prediabetic rats treated with pioglitazone, $11 \mathrm{~d}$ prediabetic rats treated with PDBT capsule. 


\subsubsection{Kidney}

Normal glomeruli and normal cellularity was found in normal rats (Figure 12-a). The prediabetic control rats (Figure 12-b) and prediabetic rats treated with standard drug (Pioglitazone) (Figure 12-c) also showed normal glomeruli and normal cellularity structure. Prediabetic rats treated with PDBT capsule (Figure 12-d) does not showed any cellular degeneration.

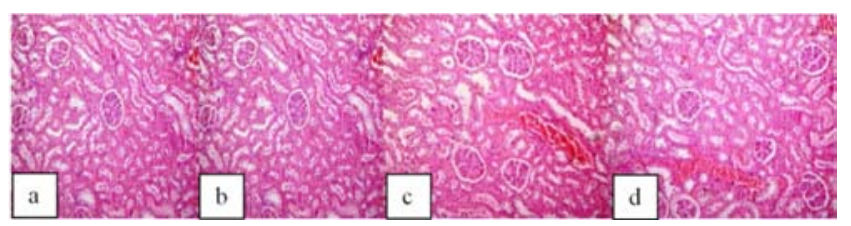

Figure 12. Histopathology study of kidney of prediabetic rats treated with PDBT capsules.

12a normal control rats, $12 \mathrm{~b}$ prediabetic control rats, $12 \mathrm{c}$ prediabetic rats treated with pioglitazone, $12 \mathrm{~d}$ prediabetic rats treated with PDBT capsule

\section{Discussion}

Knowledge of ethnopharmacology, its holistic and systems approach supported by an experiential basis from traditional Indian Medicine - Ayurveda, can serve as an innovative and powerful knowledge system for discovery of newer, safer and more affordable drugs [23].

Plants as well as herbal formulations have been used for the treatment of diabetes for several years. Here in this study the combination of extracts of well-known Ayurvedic plants was formulated as PDBT capsule. The poly herbal formulation PDBT capsule was investigated for the activity against prediabetes. Our findings indicate that treatment of $200 \mathrm{mg} / \mathrm{kg}$ of a PDBT capsule for 4-weeks in prediabetic rats led to significant improvements in several features of the prediabetes. Because of the relationship between the metabolic syndrome and prediabetes, these results have important implications for public health.

The prediabetes was induced by orally feeding high fat fructose-fructose rich diet. Several reported studies are available on induction of prediabetic condition and insulin resistance with different combinations and for different time of induction [24-26]. The selected diet mimics an unhealthy diet containing high-fat products combined with high-sugar drinks [24].

In the present study it is found that the diet combination given to the Wistar strain albino rats caused increase in body weight, fasting blood glucose levels, serum TC, TG and HDL levels and liver and kidney markers. The diet caused the increase in fasting glucose level not very high but up to prediabetes level [27]. Insulin resistance and prediabetes was confirmed by oral glucose tolerance test. The diet was enriched with fructose and fats. This resulted in obesity and insulin resistance. High fat and obesity also induce oxidative stress, this might be reason for increase in liver and kidney markers [24, 28].

The herbal extracts used formulating of PDBT capsules exert cumulative action on hyperglycemia and insulin resistance. The treatment demonstrated statistically significant decrease from $2^{\text {nd }}$ week onwards of drug intervention. At the end of $4^{\text {th }}$ week the decrease in fasting glucose was more in PDBT capsule group that of pioglitazone treatment group.

The previous study reported suggests that increase in dietary fructose could be one of the factors for obesity and the insulin resistance development [29]. Consumption of high fat fructose diet resulted in increase in body weight in all groups of animals. Obesity is one of the underlying cause of insulin resistance. Pancreas is unable to produce sufficient insulin due to obesity and which results into insulin resistance and finally type 2 diabetes [30]. Prediabetic groups when treated with PDBT capsule body weights significantly decreased as compared with HFD control group. This indicates that the PDBT capsules were effective in controlling the weight gain due to high fat fructose diet.

Consumption of cholesterol and saturated fats in diet was reflected in significant increase in cholesterol level. High amount of fructose in diet could be additional impact on lipid profile. High fructose in diet may cause increased lipogenesis and overproduction of triglycerides as well as cholesterol in hepatocytes [31]. The treatment with capsule demonstrated statistically significant decrease in elevated lipid profile. These results can be due to decrease in body weight as well as fasting glucose levels in treatment groups.

However high fat fructose diet consumption resulted in increased HDL cholesterol levels which was decreasing with with treatment. This may be due to the fact that lipid metabolism of rats is different than human [24]. Rats have strong capability to maintain their plasma cholesterol [29] additionally they do not have cholesterol-ester transfer protein (CETP) and HDL serves as major carrier of serum cholesterol [32]. The lipid metabolism in rats is primarily based on HDL rather than in human [33].

Oral feeding high fat fructose diet caused increase in the liver function markers AST and ALT, treatment of drugs decreased the elevated levels. Although the results are not statistically different this can be correlated with histopathological analysis of liver. The results show no impairment of liver cells no granular degeneration and all the samples are normal.

The consumption of large quantity of fructose caused increased blood urea nitrogen levels. The fructose diet has excessive consumption of ATP and degradation of nucleotides which results in increase blood urea nitrogen levels. The highfructose diet also impacts on serum creatinine concentration. Phosphocreatine degradation produce creatinine is produced and excreted in urine. Increase in levels of plasma creatinine if kidneys are unable to eliminate such metabolic products [34].

In the present study the kidney function markers were increased with the consumption of high fat fructose diet but not very high. In histopathological study all the samples showed no abnormality in glomeruli and normal cellularity structure. The results from histopath study and kidney function markers levels in clearly indicates that drug treatment is not disturbing the kidney functions. 
Similarly the histopathological study of pancreas also showed no inflammation and normal pancreas.

\section{Conclusion}

The results of this study indicate that PDBT capsules exhibited significant decrease in blood glucose levels on high fructose diet induced prediabetic rats. The effect of PDBT capsules on prediabetes was comparable with pioglitazone, a standard treatment for insulin resistance. The capsules resulted in improvement in hyperglycemia, dyslipidemia, obesity conditions in prediabetic rat model. The treatment did not cause any hepatotoxic, nephrotoxic and pancreatic toxic effects in rats.

Our findings suggest that, PDBT capsules may provide useful treatment for prediabetes, diabetes and related symptoms.

\section{References}

[1] Piero M, Nzaro G, Njagi J. Diabetes mellitus - a devastating metabolic disorder. Asian Journal of Biomedical and Pharmaceutical Sciences 2014; 04 (40): 1-7.

[2] Catherine C. Diabetes-Vital Statistics. USA: American Diabetes Association 1996: 65-80.

[3] Tabák A, Herder C, Rathmann W, Brunner E, Kivimäki M. Prediabetes: a high-risk state for diabetes development. Lancet 2012; 379: 2279-90.

[4] Ray J. Gestational prediabetes: a new term for early prevention? Indian J Med Res. 2010; 132: 251-255.

[5] Kristine H. Allin et al Aberrant intestinal microbiota in individuals with prediabetes Diabetologia 2018; 61: 810-820.

[6] Z. Punthakee et al. Clinical Practice Guidelines Definition, Classification and Diagnosis of Diabetes, Prediabetes and Metabolic Syndrome Can J Diabetes 2018; 42: S10-S15.

[7] Kristian Rett \& Ulrike Hostalek Understanding prediabetes: definition, prevalence, burden and treatment options for an emerging diseas, Current Medical Research and Opinion 2019; 35 (9): 1529-1534.

[8] Boyle J, Thompson T, Gregg E, Barker L, Williamson D. Projection of the year 2050 burden of diabetes in the US adult population: dynamic modeling of incidence, mortality, and prediabetes prevalence. Population Health Metrics 2010; 8: 29 .

[9] International Diabetes Federation. IDF Diabetes Atlas, 7th edn. Brussels, Belgium: International Diabetes Federation, 2015. www.diabetesatlas.org.

[10] WHO, International Diabetes Foundation. Definition and diagnosis of diabetes mellitus and intermediate hyperglycaemia: report of a WHO/IDF consultation. Geneva: World Health Organization, 2006.

[11] Rang and Dales's Pharmacology sixth edition, Churchill livingstone p. 397-409.

[12] Fabricant D, Farnsworth N. The Value of Plants Used in Traditional Medicine for Drug Discovery. Environmental Health Perspectives 2001; Suppl 1 (109): 69-75.
[13] Middha S, Mittal Y, Usha T, Dharmendra Kumar, Srinivasan $\mathrm{R}$, Vashisth L, et al. Phyto-mellitus: A phyto-chemical database for diabetes. Bioinformation 2009; 4 (2): 78-79.

[14] Tiwari A, Rao J, Diabetes mellitus and multiple therapeutic approaches of phytochemicals: Present status and future prospects. Current Science 2002; 83, (1): 30-38.

[15] Ngan T, Bao P and Ly L. Review Bioactive Compounds in Anti-Diabetic Plants: From Herbal Medicine to Modern Drug Discovery Biology 2020; 9: 252; doi: 10.3390/biology9090252.

[16] Ashok D. B. Vaidya, Thomas P. Devasagayam. Current Status of Herbal Drugs in India: An Overview J. Clin. Biochem. Nutr., 2007; 41: 1-11.

[17] Zainab M. Al-Amin, Martha Thomson, Khaled K. Al-Qattan, Riitta Peltonen-Shalaby and Muslim Ali Anti-diabetic and hypolipidaemic properties of ginger (Zingiber officinale) in streptozotocin-induced diabetic rats. British Journal of Nutrition 2006; 96: 660-666.

[18] M. Manickam, M. Ramanathan, M. A. Farboodniay Jahromi, J. P. N. Chansouria, and A. B. Ray Antihyperglycemic Activity of Phenolics from Pterocarpus marsupium J. Nat. Prod. 1997; 60: 609-610.

[19] Shanmugasundaram ER, Gopinath KL, Radha Shanmugasundaram K, Rajendran VM. Possible regeneration of the islets of langerhans in streptozotocin-diabetic rats given Gymnema sylvestre leaf extracts Journal of Ethnopharmacology 1990; 30 (3): 265-279.

[20] E Basch, S Gabardi and C Ulbricht Bitter melon (Momordica charantia): a review of efficacy and safety, American Journal of Health-System Pharmacy February 2003, 60 (4) 356-359.

[21] P. Stanely, Mainzen Prince, Venugopal P. Menon Hypoglycaemic and other related actions of Tinospora cordifolia roots in alloxan-induced diabetic rats Journal of Ethnopharmacology 2000; 70: 9-15.

[22] Takeshi O, Katsuhiro M, Takahisa Y. Pathoic physiological Changes in Pre-Diabetic Torii (SDT) Rats. Journal of Animal and Veterinary Advances 2011; 10 (7): 813-817.

[23] Pratley R, Matfin G. Pre-diabetes: clinical relevance and therapeutic approach. The British Journal of Diabetes \& Vascular Disease 2007; 7 (3S): 119-129.

[24] Patwardhan B. Ethnopharmacology and drug discovery. Journal of Ethnopharmacology 2005; 100: 50-52.

[25] Cohen M, A. Teitelbaum, and E. Rosenman. Diabetes Induced by a High Fructose Diet Metabolism1977; 26 (1): 17-24.

[26] Chinnadurai V, Mohammed A. Alsaif and Khalid S., AlNumair, Herbacetin, a flaxseed flavonoid, ameliorates high percent dietary fat induced insulin resistance and lipid accumulation through the regulation of hepatic lipid metabolizing and lipid-regulating enzymes. ChemicoBiological Interactions 2018; 288: 49-56.

[27] Russel J. C., Procter S. D. Small animal models of CVD: tool for the study of the roles of metabolic syndrome, dyslipidaemia and atherosclerosis. Cardiovascular pathology 2006; 15: 318-330.

[28] Hígado graso no alcohólico y diabetes, Non-alcoholic fatty liver disease and diabetes, Med Clin (Barc). 2017; 148 (1): $33-38$. 
[29] Rexford A. Connecting obesity, aging and diabetes. Nature Medicine 2009; 15: 996-997.

[30] Collino M, Aragno M, Castiglia S, Gianluca Miglio, Chiara Tomasinelli, Giuseppe Boccuzzi, et al. Pioglitazone improves lipid and insulin levels in overweight rats on a high cholesterol and fructose diet by decreasing hepatic inflammation. British Journal of Pharmacology 2010; 160 (8): 1892-1902.

[31] Liu I, Tzeng T, Liou S, Chang C. Angelica acutiloba Root Attenuates Insulin Resistance. Phytotherapy Research 2011; 25: $1283-1293$.
[32] Moghadasian M. H. Experimental atherosclerosis, A historical overview. Life sciences 2002; 70: 855-865.

[33] Mohammad Minhajuddin, Zafarul H. Beg, Jahangir Iqbal. Hypolipidemic and antioxidant properties of tocotrienol rich fraction isolated from rice bran oil in experimentally induced hyperlipidemic rats. Food and Chemical Toxicology 2005; 43: 747-753.

[34] Pas'koa P, Barton H, Zagrodzkia P, Gorinstein S. Effect of amaranth seeds (Amaranthus cruentus) in the diet on some biochemical parameters and essential trace elements in blood of high fructose-fed rats. Natural Product Research 2011; 25 (8): 844-849. 